\title{
Trabajo y Moralidad en las relaciones de producción capitalistas
}

BONZALO ASSUSA*

\section{Resumen}

El Trabajo como praxis ha sido ubicado, en el contexto de la modernidad, en la esfera de "lo económico", pero los discursos que lo construyen como problema tienden a revestirlo con una serie de elementos normativos y valoraciones que no se condicen con las condiciones estructurales y las "reglas de juego" propias del mercado de trabajo del sistema capitalista. Reconstruiremos aquí algunos núcleos teóricos de abordaje de la relación entre Trabajo y Cultura en términos de Moral en el marco de una regulación capitalista de las relaciones de producción. Para esto recuperaremos fragmentos de la teoría de la solidaridad social de Emile Durkheim y su relectura en la obra de Jürgen Habermas. A partir de estas discusiones, plantearemos posibles líneas de análisis en torno a las necesidades sistémicas de articulación de esferas diferenciadas como las del Trabajo y la Moral, en un mundo del trabajo instrumentalizado y fragmentado. Por último, discutiremos la necesidad de conceptualizar la relación Trabajo - Moralidad, al mismo tiempo en términos de solidaridad y disputa.

Palabras clave: Trabajo. Moralidad. Sociología del trabajo. Racionalización. Solidaridad social.

\footnotetext{
* Universidad Nacional de Córdoba (Argentina)
} 


\section{Work and Morality under capitalist relations of production}

\section{Abstract}

In the context of modernity, work as praxis has been placed in the field of Economic issues. However, the discourses that construct it as a research problem tend to attribute a set of normative elements and values that are not consistent with the structure and the "rules of the game" of the labor market in the capitalist system. In this article, we revisit some theoretical approaches to the relationship between Work and Culture in moral terms, in the context of the capitalist regulation of the relations of production. To this end, we take some elements of Emile Durkheim's social solidarity theory and its rereading in the work of Jürgen Habermas. Based on these, we propose possible analytical approaches to the systemic needs for integration between distinct fields such as Labor and Moral, considering a fragmented and exploitative labor world. Finally, we discuss the need to conceptualize the relationship between Work and Morality both in terms of solidarity and of contention.

Keywords: Work. Morality. Sociology of labor. Rationalization. Social Solidarity.

\section{Introducción ${ }^{1}$}

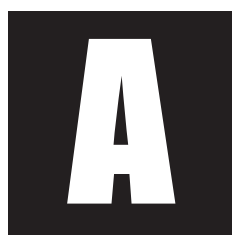

I abordar la problemática del empleo en general, y del empleo juvenil en particular, el investigador se encuentra con un entramado de discursos que operan sobre la problemática en una clave de moralidad. En cierta forma, esto constituye una paradoja: mientras que el trabajo se ubica, en el contexto de la modernidad, en la esfera de "lo económico", los discursos que lo construyen como problema tienden a revestirlo con una serie de elementos normativos y valoraciones que no

\footnotetext{
${ }^{1}$ Agradezco la generosidad de las lecturas, observaciones y críticas que Álvaro Michelli y Juan Manuel Barri hicieron sobre la primera versión de este texto.
} 
se condicen con las condiciones estructurales y las reglas de juego propias del mercado de trabajo del sistema capitalista.

El Trabajo como praxis humana ha estado en el centro de los debates sobre el proceso de racionalización en el mundo moderno y ha estructurado la inteligibilidad de la sociedad como Totalidad (Lukacs, 1985). Sin embargo, precisamente por su posición central, la configuración moderna del trabajo ha dado lugar a una serie de (funcionales) confusiones en torno a la univocidad instrumental y estratégica que lo inviste desde el advenimiento de la razón moderna ¿Bajo qué criterios podemos entender que el trabajo como acción está escindido [absolutamente] del carácter cosmológico y moral, y que se orienta [exclusivamente] por una racionalidad de tipo instrumental?

Este problema puede observarse, en la práctica, en numerosos «reclamos» morales en torno a una relación que, tanto la economía política como la teoría crítica marxista (aunque, desde lugares opuestos) han definido como "libre" de cargas personales y políticas: situada en el mercado (laboral), terreno desembarazado, por antonomasia, de contenido normativo. Sin embargo, la práctica laboral no dejó de funcionar, a lo largo del tiempo, como el mérito necesario para el acceso a la dignidad personal del individuo moderno, para el prestigio social y la ciudadanía ¿Cuáles son, entonces, los vínculos que unen a la categoría sociológica de trabajo con los repertorios culturales y morales modernos?

En lo que respecta al vínculo entre estas categorías, hemos podido rastrear algunas reseñas y estados del arte que hacen un recuento bastante exhaustivo de las distintas aproximaciones a los aspectos simbólicos del mundo del trabajo que han realizado los estudios laborales y la sociología del trabajo (Drolas, Lenguita, Montes Cató y Wilkis, 2009; Guadamarra Olivera, 2000; Palenzuela, 1995). Por nuestra parte, entendemos que, de distintas maneras y con distintos nombres, el vínculo entre Trabajo y Cultura (como forma genérica de nominar las relaciones y los sistemas simbólicos) ha sido, antes que una temática sub-disciplinar, un núcleo 
teórico desde el cual la sociología clásica comprendió procesos más allá y más acá de la realidad - estrictamente- laboral.

Aquí, el retorno sobre el tratamiento de un tema en su versión clásica no tiene una finalidad puramente "arqueológica". Antes bien, adherimos al planteo de Jeffrey Alexander acerca la centralidad esencial de los clásicos para la constitución de un horizonte discursivo común en las ciencias sociales (Alexander, 1998, p. 35). Tal como lo plantea este autor, La necesidad funcional de los clásicos se origina en la necesidad de integrar el campo del discurso teórico (Alexander, 1998, p. 42), de "reducir la contingencia", simplificar y facilitar la discusión teórica, además de operar sobre los conceptos construidos en el discurso científico la misma vocación de vigilancia e historización que sobre las categorías del sentido común.

De este modo, el presente texto apunta a reconstruir algunos núcleos teóricos de abordaje de la relación entre Trabajo y Cultura en términos de Moral en el marco de una regulación capitalista de las relaciones de producción ¿Cómo es que el trabajo puede movilizar diferencias culturales y morales habiéndose desanclado, en el mundo moderno occidental, de motivaciones normativas? La obra de Emile Durkheim aún hecha luz sobre procesos problemáticos contemporáneos. Este autor centra sus preocupaciones en el ámbito de la moralidad, a partir de lo cual permite pensar el trabajo en un sentido no-restringido a su dimensión técnico-instrumental (bajo la noción de "trabajo social"). Por otro lado, aún con los rudimentos de su teoría, establece una conexión entre las transformaciones en el medio social (estructura y funciones) y la mutación morfológica de lo que, en estadios sociales "menos desarrollados", podía denominarse como "conciencia colectiva".

Intentaremos reconstruir algunos de sus argumentos para pensar la formación social capitalista, aunque este no haya sido el objetivo conceptual específico de este autor. Consideramos que esto no quita la posibilidad de hacer dialogar las lecturas de Emile Durkheim con algunos desarrollos teóricos marxianos, de una manera que trascienda la contraposición más clásica entre paradigmas disímiles. En algún sentido, la reconstrucción de la 
preocupación de estos autores por las tendencias de desarrollo de la sociedad nos fue más útil para reevaluar sus aportes en pos de pensar el vínculo problemático entre las categorías de «trabajo» y «cultura».

Con este fin, recuperaremos algunas lecturas de Jürgen Habermas sobre la teoría de la solidaridad social y sus análisis a partir de la estrategia conceptual de postular la sociedad en términos de sistema y mundo de la vida, enfocando nuestra atención específicamente en el debate sobre la generación de contenidos normativos desde ámbitos, como el de Trabajo, regidos por una lógica instrumental des-lingüistizada.

A partir de esto, el texto pretende evaluar la posibilidad (y la necesidad) de pensar sociológicamente un análisis de las relaciones entre trabajo y cultura (en términos de moral) en el marco de las relaciones capitalistas de producción. En este contexto, plantearemos algunas líneas teórico-conceptuales de reflexión de la relación trabajo-moral, para el análisis de las relaciones laborales contemporáneas en torno a las discusiones teóricas que nos damos en el texto. La búsqueda y el esfuerzo de cotejamiento conceptual apuntan a una operación de desnaturalización típica de las ciencias sociales: reconstruir los procesos por los cuales el trabajo y la cultura se constituyeron como esferas -relativamente independientes- de la realidad propiamente social, y, en el mismo acto, mostrar las múltiples conexiones, rearticulaciones y re-acoplamientos entre estas esferas: la necesidad de hilos conductores y lazos -entre el ámbito de la producción material y el ámbito de la producción simbólica²- para la reproducción de la formación social en general, y de las relaciones laborales en particular, en términos de Totalidad.

\footnotetext{
${ }^{2}$ Como mostraremos a lo largo del texto, nuestro argumento no adscribe al pensamiento de estas como instancias efectivamente separadas en su sustancia, aunque su distinción analítica haya servido al menos para dos fines fundamentales: 1) reconocer no ya su separación esencial, sino las condiciones sociales necesarias para su autonomización, y 2) comenzar a pensar la forma específica en la que estas instancias se articulan.
} 
Finalmente, discutiremos las posibilidades de análisis abiertas por estos desarrollos conceptuales, y la necesidad de construir teóricamente la relación trabajo-moralidad en términos de solidaridad y regulación social, pero también en términos de recurso, disputa y producción de desigualdad y diferencia.

\section{Contexto histórico: la des-personalización de las relaciones de producción}

En un principio, fue la comunidad. Tal como lo muestra Robert Castel (2000), la problemática del socorro ligada a la cuestión del trabajo tiene una larga historia en el occidente europeo. Sin embargo, mientras que en la pre-modernidad la cohesión social se aseguraba en términos de territorialidad restringida y de sociabilidad primaria, a medida que la lógica del mercado se fue imponiendo en la dinámica económica, nuevas experiencias de solidaridad se fueron haciendo necesarias. De esta manera, llegamos al contexto que nos describe Habermas como Capitalismo liberal: una formación social con sistemas político y económico desanclados entre sí, con relaciones de clase despolitizadas y con poder de clase anonimizado (Habermas, 2002, p. 43).

En este marco se fue generando una regulación primero moral (la asistencia ligada a la religión y sus instituciones), y luego política (vinculada al Estado de Bienestar europeo continental) de la relación salarial. Pues bien, al liberar la producción material de las regulaciones tradicionales y de la dependencia personal impuesta en marcos comunitarios, la regulación capitalista de la producción se generó un cuerpo de "protecciones sociales" inventando, en términos de Castel, la "Cuestión social" como dispositivo que clasifica y constituye los grupos de la sociedad. La manera que el sistema encontró para garantizar la cohesión social en el marco 
de relaciones sociales de producción des-personalizadas fue a partir de los medios burocrático-administrativos del Estado y de la constitución del salariado como estatuto, es decir, reconfigurando el conflicto social Capital-Trabajo en términos de "competencia". Sin embargo, al tener en cuenta la solución histórica que Castel reconstruye para el problema de la cohesión social, surge la pregunta: ¿Dónde quedó el contenido normativo? Evidentemente, este substrato no se encuentra en gran medida ni en la burocracia estatal, ni en la individualización del salariado.

A partir de esta problemática, intentaremos vincular la teoría de la solidaridad de Emile Durkheim con estos dos aspectos del proceso de salarización del trabajo y con sus consecuencias: primero, con la progresiva des-personalización de las relaciones de producción en el Capitalismo; y luego, con la necesidad de re-moralización y re-politización de la producción para garantizar la reproducción del sistema.

\section{División del trabajo y solidaridad social}

En La división del trabajo social y en El suicidio Durkheim reflexiona sobre el creciente nivel de conflictividad en las sociedades industriales de su época a partir del análisis acerca del déficit en los mecanismos de integración social. No nos centraremos tanto aquí en la discusión acerca de la explicación de la causa de la división del trabajo como hecho social, sino que intentaremos reconstruir lo que el autor identifica como una tendencia de desarrollo que fue recuperada en cuerpos teóricos más contemporáneos, renombrada y reelaborada: como diferenciación funcionalsistémica, en Parsons, y como autonomización de subsistemas de acción desanclados de contenido normativo, en Habermas.

Sí importa señalar que, para Durkheim, estas transformaciones en el "medio social" (la división del trabajo) tienen consecuencias concretas en 
el ámbito de la "moralidad". Si bien Durkheim no habla de "cultura" en el mismo sentido en que muchas veces suele usarse con posterioridad a la discusión y al aporte específicamente antropológico al término, su noción de "moral" está emparentada en gran medida con la forma en la que Parsons pensaría su noción de sistema (o sub-sistema) cultural. Compuesto de normas y valores, "patrones" que sirven para la orientación estable de la acción de los actores-en-rol del sistema social ${ }^{3}$. Para Durkheim, la moral [...] comprende todas las reglas de acción que se imponen imperativamente a la conducta y a las cuales está ligada una sanción [...] (Durkheim, 1985, p. 62).

La distinción durkheimiana entre distintos tipos de estructura social (sociedades segmentadas u orgánicas) constituye un ingreso en detalle sobre el modo en el que el autor considera que opera el ámbito de la moralidad. Si la "conciencia colectiva" podía ser entendida como una configuración concreta de la dimensión moral, en tanto sentimientos y creencias compartidas por los miembros de una sociedad (Durkheim, 1985, p. 94), es también cierto que ésta definía y estructuraba las "conciencias individuales", sus percepciones $(y, \text { podemos pensar, sus acciones })^{4}$. Hasta aquí, la problemática del vínculo trabajo-moral no llega a plantearse, justamente porque el análisis se sitúa en un particular momento histórico en el que prima el estado de identidad entre la conciencia individual y la conciencia colectiva.

El problema específicamente moral surge con el advenimiento de un sistema de funciones diferenciadas y especializadas que se ve acompañado por un progresivo desdibujamiento de la conciencia colectiva. La ruptura de la identidad entre las conciencias individual y colectiva no

\footnotetext{
${ }^{3}$ La cultura se asimila a la función de latencia. Ella proporciona a los actores los elementos de motivación y el soporte de la acción, por las normas, ideales, valores, ideologías que propone o les impone (Rocher, 1972, p. 22).

${ }^{4}$ Así planteadas las cosas, no habría razón suficiente para leer la relación Sociedad - Individuo en Durkheim como una relación entre "polos", sino más bien como una relación de constitución, en una configuración social particular en la que estos dos momentos se hallaban en coincidencia.
} 
dejaría, sin embargo, un vacío tras de sí: generaría una evolución social de base profesional, con una coordinación y subordinación específica, con una moralidad intrínseca en desarrollo (Durkheim, 1985, p. 226) ${ }^{5}$.

Para Durkheim, la división del trabajo, en tanto mecanismo evolutivo, no produce disgregación. Antes bien, genera un aumento de la densidad moral y material de la sociedad (acompañado, por ley general, de un aumento en el volumen social). En este sentido, tal como lo entiende Durkheim, este mecanismo debe producir sus formas específicas de reciprocidad (Durkheim, 1985, p. 214) ${ }^{6}$.

Para Durkheim, el trabajo como práctica no era exclusivamente un medio técnico de aumento de rendimiento (aunque no dejara de serlo), sino, sobre todo, una fuente fundamental de solidaridad, basada y estabilizada por valores como el "mérito". Las disrupciones, en este esquema, vienen dadas por situaciones de anomalía y no son consustanciales al proceso evolutivo: surgen por la ruptura de la espontaneidad en la división del trabajo, el desarreglo de las expectativas y las recompensas, el uso de la violencia, etc.

\footnotetext{
${ }^{5}$ El prólogo a la segunda edición de La división del trabajo social hace alusión a este planteo: Se trata del papel que las agrupaciones profesionales están destinadas a llenar en la organización social de los pueblos contemporáneos (Durkheim, 1985, p. 1). En otros textos, esta preocupación por las corporaciones y su lugar en la organización social, reaparece: [...] la sociedad está demasiado lejos de los intereses especiales que se trata de regular, de los antagonismos que se trata de apaciguar, para poder desempeñar ese papel moderador, sea por sí misma o por medio de los poderes públicos. Por eso le interesa dejar que los grupos particulares se constituyan y se encarguen de esa función (Durkheim, 2003, p. 87). Algunas de las argumentaciones de El suicidio van en esa dirección también (Durkheim, 1994).

${ }^{6}$ Nótese que otros autores también trabajaron bajo el supuesto de la existencia de una moralidad específicamente moderna. Es el caso, por ejemplo, de Norbert Elías: Pero la diferenciación progresiva de las funciones sociales no es más que la primera y más general de las transformaciones sociales que se ofrecen a la consideración del observador cuando éste investiga las causas de los cambios de los hábitos psíquicos que impone una «civilización». Paralelamente a la diferenciación, a la progresiva división de funciones, se produce una reorganización total del entramado social (Elías, 1989, p. 453). Sin embargo, el avance de este autor fue la incoporación de la dimensión de la estructura subjetiva en su análisis, junto con el reconocimiento de esta doble transformación de las configuraciones sociales: división funcional y aumento de la interdependencia (lazos, a la vez, sociales, económicos y simbólicos).
} 
De esta manera, al pensar el trabajo como "función"7 (de constitución de solidaridad), Durkheim no concibe ninguna contradicción en relación a la esfera de la moralidad. Sería injusto para el autor plantear que en su esquema no existe lugar alguno para la estratificación, las agrupaciones o hasta las clases sociales. Su perspectiva hace justamente foco en las corporaciones (en un sentido descriptivo-normativo), mientras que lo que deja de lado es la tematización específica de las "relaciones de explotación" (producción y apropiación de excedente). Aunque esta aclaración parezca una obviedad, brinda la clave para comprender que la división del trabajo social, en tanto proceso de civilización, no es comprendido como un proceso de "des-moralización", sino más bien como un proceso de "fragmentación-multiplicación-diferenciación moral".

Si pensamos este proceso de civilización en paralelo al proceso de formación social capitalista, podemos identificar ciertas innovaciones conceptuales atribuibles a Durkheim. Marx, por su parte, reconstruye la transformación en las relaciones de producción desde el modo de producción feudal al modo de producción capitalista como un despojamiento del carácter "personal" y "político" de esta relación".

Es necesario que sea superada esta apariencia; que la propiedad de la tierra, raíz de la propiedad privada, se comprometa en el movimiento de la propiedad privada y se

\footnotetext{
${ }_{7}$ Podríamos complejizar esta idea a partir de la distinción introducida por Merton (1980, p. 156) entre función manifiesta y función latente. Mientras que encontramos el sentido instrumental atribuido al trabajo en el discurso de la economía política, Durkheim adjudica la función latente de creación de lazos de solidaridad, para lo cual invertirá buena parte de su argumentación en La división del trabajo social.

${ }^{8}$ Algunos autores marxistas señalaban los problemas que acarrea la separación -propia del capitalismo- de las categorías de "lo político" y "lo económico": Esta separación conceptual, si bien refleja una realidad específica del capitalismo, no sólo no comprende las muy diferentes realidades de las sociedades precapitalistas o no capitalistas, sino que también disfraza las nuevas formas de poder y dominación creadas por el capitalismo (Meiksins Wood, 2000, p. 16). Luego volveremos sobre este tema.
} 
convierta en mercancía; que la dominación del propietario aparezca como la dominación pura de la propiedad privada, del capital, desprovista de todo tinte político; que la relación entre propietario y trabajador se reduzca a la relación económico-política entre explotador y explotado; que cese toda relación personal de propietario con su propiedad y que esta misma se convierta en riqueza objetiva y material (Marx, 2004, p. 99. La cursiva es mía).

Sobre lo mismo, arguye Habermas,

Y puesto que la coacción social ejercida por los capitalistas se ha institucionalizado en el contrato de trabajo privado como relación de intercambio, y la extracción de la plusvalía, sobre la que disponen los particulares, ha reemplazado a la dependencia política, el mercado, además de su función cibernética, adquiere una función ideológica: la relación de clases se vuelve anónima en la forma no política de la dependencia salarial (Habermas, 2002, p. 49).

Es por esto que, al fundar una relación de tipo "exclusivamente económica", la economía política puede ocultar el carácter ideológico de una relación (de explotación) que se presenta como un intercambio entre equivalentes (producción de valor y salario), que no son tales. La categoría de "plusvalía" pone en evidencia concretamente esta diferencia. Durkheim está pensando de otra manera. En primer lugar, la diferenciación funcional no se instancia exclusivamente en el ámbito de la producción material ${ }^{9}$. El proceso incluye especialización en cuanto a fun-

\footnotetext{
${ }^{9}$ Esto no quiere decir que para Marx esto suceda exclusivamente en el ámbito de la economía. Pero ciertamente, no sólo su centro, sino también su punto de partida se ubica en el modo en el que los hombres organizan la producción material de su vida: La primera premisa de toda historia humana es, naturalmente, la existencia de individuos humanos vivientes. El primer estado que cabe constatar es la organización corpórea de estos individuos, y como consecuencia de ello, su relación con el resto de la naturaleza [...] Los individuos tal y como manifiestan su vida. Lo que son coincide, por consiguiente, con su producción, tanto con lo que producen como con el modo de cómo producen. Lo que los individuos son depende, por tanto, de las condiciones materiales de su producción (Marx; Engels, 2004, p 11-12).
} 
ciones políticas, funciones religiosas, y en todo lo que pueda incluirse en la noción de "trabajo social" [es decir, socialmente necesario para la reproducción de la sociedad]. Por otra parte, al no haber una tematización de la desigual apropiación del excedente, el proceso de racionalización aparece en un sentido casi exclusivamente horizontal: como sistema de funciones especializadas. El proceso de "racionalización" de las relaciones de explotación, que las despoja de contenido político (aquel que vinculaba al amo y al esclavo, al señor y al ciervo) se observa sólo al tematizar el sentido vertical de estas relaciones: como relaciones de explotación, entre apropiadores del producto y productores directos. Justamente, al pensar procesos de desigualdad (en las sociedades primitivas) Durkheim distingue la relación cosificada de una relación signada por la reciprocidad:

Sin embargo, el lazo que, en ese caso, une al individuo al jefe es idéntico al que en nuestros días liga a la cosa a la persona. Las relaciones del déspota bárbaro con sus súbditos, como las del dueño con sus esclavos, del padre de familia romana con sus descendientes, no se distinguen de las del propietario con el objeto que posee. No tienen nada de esta reciprocidad que produce la división del trabajo (Durkheim, 1985, p. 214).

La dominación política o económica (explotación, extracción de plusvalía) no aparece en el núcleo central de su lectura del proceso de división del trabajo. La manera en la que Durkheim plantea su problema lleva a pensar en el carácter personal o político (en el lenguaje que estamos utilizando, moral) de relaciones entre funciones específicas y entre elementos de la estructura social modificada a partir de la fusión de segmentos. Por esto también podemos comprender que, en el pensamiento de este autor, la conflictividad social se configure como una "tendencia centrífuga" (déficit de integración) antes que como una "contradicción" (dialéctica), es decir, como una "crisis", como déficit de racionalidad. 


\section{El trabajo y la interacción: el contenido normativo en sentido restringido}

Para dar cuenta de la manera en que la necesidad de contenido normativo y motivacional se resuelven en un proceso de diferenciación estructural, falta un paso, y es el de distinguir entre trabajo e interacción: Ciertamente que las reglas técnicas sólo se forman bajo las condiciones de la comunicación lingüística, pero no tienen nada en común con las reglas comunicativas de la interacción (Habermas, 1999, p. 36). En un giro hegeliano, Habermas entiende que estos dos ámbitos de la acción son irreductibles entre sí. En este sentido retoma, en el tomo II de la Teoría de la acción comunicativa, la teoría de la solidaridad social de Durkheim como un insumo necesario para el desarrollo de una estrategia conceptual doble para el abordaje de la sociedad: en términos de sistema y de mundo de la vida. En este proceso, la reconstrucción que Habermas opera sobre los desarrollos de Durkheim, a través del tamiz de una teoría de la modernización con eje en la racionalidad, que va desde Marx, pasando por Weber, hasta Lukács y la Teoría Crítica, aporta elementos importantes para pensar los núcleos problemáticos del vínculo trabajo-moral específicamente en el marco de relaciones de producción de la formación social capitalista contemporánea.

Este autor intenta dar cuenta del problema de la racionalización como problema sociológico, en dos sentidos: como proceso de "desencantamiento del mundo" -en tanto visión de la sociedad sobre sí misma-, y como instalación de la "racionalidad instrumental" como eje de la interacción humana ${ }^{10}$. Si bien no tenemos aquí el espacio para plantear una reconstrucción del camino teórico de este autor, a fin de abordar el vínculo tendido entre trabajo y moral, deberíamos establecer que, en su relectura de

\footnotetext{
${ }^{10}$ Para ver una discusión detallada de las características del plan de trabajo de Jürgen Habermas, ver el trabajo de Axel Honeth (1998).
} 
Marx, Habermas distingue en su teoría de la sociedad dos tipos de acción -subsumidas por el marxismo bajo la lógicoa técnico-productiva- que, según entiende, son necesarios para la estructuración de las interacciones y para la reproducción de la sociedad: por un lado, el trabajo, homologado a la acción estratégica orientada a fines; y por el otro, la interacción, como acción comunicativa orientada al entendimiento ${ }^{11}$. Es en este sentido que Habermas decide basar su análisis de la sociedad en una analítica de la comunicación. De una manera simplificada, este es el punto de partida de su razonamiento: El trabajo o la acción instrumental se rige por reglas técnicas; estas encarnan supuestos empíricos que implican pretensiones de verdad, es decir, pretensiones de validez corroborables discursivamente y sujetas por principio a la crítica (Habermas, 2002, p. 28).

Ahora bien, ¿Cómo vincular la reproducción material de la sociedad en la configuración de «sistemas», y la reproducción simbólica del «mundo de la vida» (intersubjetividad como trasfondo común de la comunicación)? A partir de este núcleo problemático es que Habermas vuelve sobre la obra de Emile Durkheim y su teoría de la solidaridad social como análisis de la integración.

Tal como Habermas lo entiende, Durkheim lee el proceso de modernización como un cambio en las bases de la integración social, desde un consenso normativo básico hacia una conexión sistémica de ámbitos de acción funcionalmente especificados (Habermas, 1990, p. 163). Al reconstruir la discusión entre Spencer y Durkheim, Habermas encuentra

\footnotetext{
${ }^{11}$ Así Marx, sin tener conocimiento de los manuscritos de Jena, redescubre en la dialéctica de fuerzas productivas y relaciones de producción esa conexión de trabajo e interacción [...] El mecanismo de mutación del sistema del trabajo social lo encuentra en la contradicción entre el poder de control sobre los procesos naturales acumulado por el trabajo y el marco institucional de interacciones reguladas de forma espontánea y no reflexiva [...] Marx no explica en realidad la conexión existente entre trabajo e interacción, sino que bajo el rótulo inespecífico de práctica social reduce lo uno a lo otro, es decir, la acción comunicativa a la instrumental (Habermas, 1999, p. 49).
} 
que, mientras que el primero pensaba en la división del trabajo como un mecanismo de integración no-normativo, Durkheim entendía que

También la forma orgánica de solidaridad social tiene que venir asegurada por medio de normas y valores; sigue siendo, lo mismo que la mecánica, expresión de una conciencia colectiva, aunque se trate de una conciencia colectiva transformada en sus estructuras (Habermas, 1990, p. 165).

Luego de su reconceptualización y de la incorporación de Durkheim en su esquema de reconstrucción de la historia de la sociología, Habermas plantea que el análisis de la problemática de la integración por parte de Durkheim puede ser entendida como una "crítica de la cultura burguesa", en la medida en que deduce las patologías de la sociedad a partir de la racionalización misma del mundo de la vida (la esferización del mundo y un ámbito cada vez mayor de la acción librado a la reflexividad del individuo), desvinculando la problemática respecto de la reproducción material del mundo de la vida (a diferencia de la manera en que Marx había entendido el mismo proceso). La "anomia", entonces, surge como una disrupción en un mundo de la vida con elevada diferenciación estructural, mientras que la solidaridad orgánica aparecería como la forma normal de integración social en una sociedad con un mundo de la vida racionalizado.

En este sentido Habermas replantea el problema de la cosificación a partir del registro de la autonomización de subsistemas de acción regidos por medios de control des-lingüistizados (Habermas, 1990), esto es, la colonización del mundo de la vida por parte del sistema, o bien, la instrumentalización de las interacciones humanas. Uno de estos medios nonormativos es el del Dinero, en tanto medio de coordinación que surge en determinado momento de diferenciación sistémica. Este constituye el único elemento "institucionalizado" en el sistema económico, en el cual representa un fragmento de sociabilidad exento de contenido normativo. En sus propias palabras, [...] la empresa capitalista y la administración 
moderna son unidades sistémicamente autonomizadas en el seno de subsistemas exentos de contenido normativo (Habermas, 1990, p. 243).

Reconstruir el proyecto teórico general de Habermas aparece como central para pensar nuestra relación problemática. Justamente, el esfuerzo hecho por el autor intenta reconectar lo que el proceso de modernización muestra como separado, tendiendo puentes entre lo que la modernidad ha construido como mundos autónomos: por un lado, el de la economía y la técnica, y por el otro, el de la cultura y la moral. Habermas reubica la cuestión de la diferenciación funcional (de la reproducción material) en un ámbito específicamente económico (aunque su tematización incluye en gran parte el análisis de la evolución del sistema político). En el fondo, la relectura de Habermas permite desplazar la pregunta hacia el seno de la contradicción de la formación social capitalista: ¿cómo (re-)acopla el sistema capitalista una economía tecnificada y orientada por la racionalidad instrumental, y por lo tanto, una regulación "exclusivamente económica" de las relaciones laborales, con una necesaria integración social que sirva como sustrato de entendimiento básico para la estructuración de la interacción ? $^{12}$ Incluso en el ámbito de la sociedad capitalista, la reproducción del sistema no se da exclusivamente en términos mecánicos, sino que requiere insumos de legitimidad, identidad, entendimiento, etc. Incluso si se deja de lado el arsenal conceptual de la teoría de la acción comunicativa, la mayor parte de las teorías sociales contemporáneas in-

\footnotetext{
${ }^{12}$ Esto genera un problema adicional, y que no podremos aquí sino sólo dejar planteado. El artificio teórico de Habermas tiene, a la vez, la virtud y el defecto de resultar esquemático, y de quedar, en cierta forma, acoplado al discurso moderno sobre la sociedad: "Trabajo" e "Interacción" aparecen como formas de acción radicalmente diferenciados, lo cual plantea algunos problemas empíricos en el análisis de las prácticas laborales. Tal como lo plantean Eyerman y Shipway, el tiempo de trabajo, para el trabajador, no sólo lo determina objetivamente en tanto mercancía, sino también como sujeto, como ser humano (1981, p. 562). Tal como ellos sostienen, aunque la dimensión cultural (personal y política) del proceso de trabajo se ve restringida, sigue presente y no se ha instrumentalizado simplemente en un proceso patológico, sino que mantiene aún su carácter interactivo. En este sentido, creemos que debería explorarse la posibilidad de una conceptualización del trabajo como práctica esencialmente ambivalente.
} 
tentan dar cuenta de la búsqueda de reconocimiento por parte de los agentes, de las suturas ideológicas, los mecanismos de estabilización de la experiencia, de la generación hegemónica de consenso o de la definición común de la situación. Se reconoce entonces, la necesidad de considerar el sentido como aspecto constitutivo incluso de aquellas acciones reguladas por ámbitos, reglas y lógicas técnico-instrumentales.

Dicho todo esto, ¿Puede existir, entonces, una moral o una cultura del trabajo en su condición salariada, capitalista, alienada?

\section{Re-acoplamiento de la moral y el trabajo en contextos de fragmentación e instrumentalización}

Uno de los problemas teóricos más fuertes asociados al análisis de la articulación de estas instancias es que, en el marco de las discusiones subdisciplinares de la sociología del trabajo, las fuertes transformaciones en el mundo del trabajo que se registraron a partir de los años setenta generaron, o bien una puesta en cuestión radical de la centralidad del trabajo para explicar la estructuración de lo social, o bien una absoluta fragmentación, disgregación y multiplicación del pensamiento de la cultura y la moral en torno a lo laboral (De la Garza Toledo, 2000). Sin negar las transformaciones tendientes en las últimas décadas a una mayor diferenciación, fragmentación y diversificación de las prácticas en el mundo de la producción, sumado a una emergencia mucho más visible y problemática de la cuestión del desempleo y el subempleo en gran parte del mundo occidental ${ }^{13}$, entendemos que dichos virajes

\footnotetext{
${ }^{13}$ Tal como sostiene Martín Criado, A partir de los años 70 una serie de dinámicas -en parte fruto de la crisis económica las políticas que se comienzan a poner en marcha- alteran de manera profunda la estructuración del mercado de trabajo. Estas transformaciones socavan progresivamente la delimitación de fronteras nítidas entre las situaciones de paro, empleo e inactividad (Martín Criado, 1999, p. 28).
} 
no invalidan lo argumentado acerca de la centralidad de las articulaciones entre esferas para la reproducción social.

Lo cierto es que algunos equívocos conceptuales contemporáneos llevaron a invalidar la pregunta por esta articulación, o a conducirla, desde una perspectiva fragmentada, a un callejón sin salida. Uno de estos equívocos es el de asimilar los procesos identitarios a los procesos culturales en general (Grimson, 2011). Mientras que muchas de las objeciones a las perspectivas centradas en la categoría «trabajo» para el análisis de la realidad social señalaban una transformación en las formas de agrupamiento y articulación de acciones colectivas, ya no determinadas por las posiciones en las relaciones de producción, lo que se perdió de vista es que, incluso cediendo a este argumento teórico, no había razón para deducir de esto una consiguiente pérdida de poder del constructo moral en torno al trabajo como principal instancia de construcción de dignidad personal y de legitimidad política, es decir, de articulación hegemónica del horizonte de significaciones culturales posibles.

El segundo equívoco, profundamente vinculado con el anterior, es el supuesto de que la fragmentación de prácticas, posiciones y procesos de trabajo, y la precarización e inestabilización de las vidas y las trayectorias laborales volvía estructuralmente inviables procesos simbólicos que pudiesen reconstruirse con cierta unicidad (Sennett, 2000; Bauman, 1999). Algunas de estas dificultades fueron supuestamente resueltas con el gesto de tornar plurales las categorías otrora singulares: de "cultura" a "culturas" del trabajo. La salida más radical, asociada al relato del "fin del trabajo", optó por desestimar de plano la indagación por estas articulaciones, observando que los elementos más relevantes de las construcciones simbólicas pasaban por fuera del ámbito de la producción material (y con autonomía respecto de éste). 
Sin embargo, algunos de los trabajos ya clásicos sobre los aspectos simbólicos del proceso de producción capitalista y las transformaciones que propone en la experiencia de la clase trabajadora (Thompson, 1989), mostró que esta mutación se dio a partir de una serie de instancias y acciones institucionales, organizacionales y disciplinares (religión, barrio, sociedades de fomento, clubes de lectura, fábricas, etc.), que condicionó una formación de clase [procesual] obrera, pero que nunca apareció históricamente como una experiencia del trabajo ya unificada desde el primer momento.

Lo que de alguna manera queremos señalar es que las transformaciones generadas en las últimas cuatro décadas en torno al mundo del trabajo revitalizan, antes que anulan, la pregunta por los hilos conductores entre las esferas del trabajo y de la moral. La actual configuración de las relaciones en el mundo del trabajo, con todos los adjetivos que desde los estudios laborales le han sido atribuidos - inestables, precarias, interrumpidas, discontinuadas, etc. - mantienen absolutamente vigente la necesidad estructural de un re-acoplamiento político de las esferas que la modernidad autonomizó: programas, intervenciones, políticas, estatales y no gubernamentales, por dentro y por fuera del ámbito y los espacios laborales, regulan e intervienen las prácticas, fundamentalmente de aquellos sectores más afectados por la inestabilidad y los problemas de empleo.

Estas intervenciones no se limitan exclusivamente a la cuestión "técnica" de capacitación de la fuerza de trabajo para su ingreso en el mercado laboral (Jacinto y Millenaar, 2012; Neffa, Brown y López, 2012). En los últimos años, adquirieron una importancia fundamental aquellas intervenciones que se dieron a la tarea de "orientar", "inducir" y "formar" en las normas de interacción en el mundo del trabajo, a reinstalar el trabajo como "valor" en la vida de los sectores subalternos, a moralizar las trayectorias a partir de la estabilización de las prácticas laborales, pero 
fundamentalmente, a reforzar al trabajo como instancia, por excelencia, con capacidad de legitimar la asignación de recursos monetarios ${ }^{14}$.

En este sentido, entendemos que la necesidad estructural de re-acoplamiento trabajo-moral, disparada por los procesos de racionalización, autonomización y des-normativización, sigue vigente incluso en un mundo en el que el trabajo (y la economía en general) está más que nunca signada por una instrumentalización exacerbada. Es en este contexto donde aparecen con más potencia una serie de instancias e intervenciones que tienden a borrar la nitidez de las fronteras entre los problemas "técni-

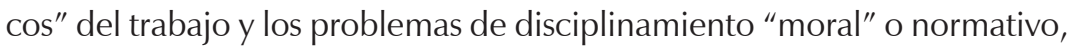
entre capacitación en competencias y la formación actitudinal, entre el proceso de trabajo y el mercado de trabajo, entre lo simbólico y lo instrumental en el mundo del trabajo ${ }^{15}$.

\section{Palabras finales: Moral, Trabajo y Política en las sociedades capitalistas}

Volviendo a la manera en la que Durkheim piensa las transformaciones en la conciencia colectiva, podemos plantear que las configuraciones de la moralidad emergen, según el autor, del devenir y las mutaciones del medio social - entendido como estructura social -, aunque a su vez éstas reactúen y regulen las acciones de los individuos, instituyendo y dotando de sentido determinadas necesidades funcionales de la sociedad.

\footnotetext{
${ }^{14}$ Para un estudio etnográfico de los componentes morales de la circulación de dinero y mercancías en una serie de políticas, organizaciones y prácticas filantrópicas, ver Wilkis y Carenzo (2008).

${ }^{15}$ El presente trabajo se enmarca en un análisis más amplio sobre esta serie de instancias y su comprensión en el marco de los procesos de reproducción social de jóvenes en un barrio popular del conglomerado urbano de Gran Córdoba. Aquí sólo presentamos lo que consideramos una necesaria puesta a punto conceptual que recupere, teóricamente, la perspectiva de la totalidad y construya una noción de moral y cultura del trabajo como un proceso simultáneamente material y simbólico, atravesado por relaciones de dominación de clase y de dependencia personal.
} 
De esta forma, y por su análisis de determinadas problemáticas culturales vinculadas al trabajo - como la sensación de "des-humanización" de la época, la "frustración" y la construcción de criterios "meritocráticos" para la regulación de la acción social - Durkheim otorga entidad a dos dimensiones que necesariamente deben abordarse [conjuntamente] para la problemática del trabajo: la técnico-funcional y la moral-cultural.

Respecto de esta segunda dimensión, no correspondería hablar de una "autonomía relativa", pero de seguro la moral no aparece como mero reflejo de las mutaciones en la estructura de las sociedades. Sus configuraciones (la conciencia colectiva y las diversas morales profesionales que las corporaciones debían constituir y consolidar) regulan y estructuran las acciones de los individuos, y en este sentido, tienen peso propio como objetos de indagación.

Por otra parte, en base a las diferencias entre este autor y Spencer, para Durkheim el crecimiento de la división del trabajo social no se ve como el paso de una "integración social normativa" (solidaridad mecánica) a una "coordinación no-normativa de la acción por plexos funcionales específicos" (solidaridad orgánica), sino que reconoce que la división del trabajo posee (o debería poseer) una moralidad intrínseca. Con ello, abre la posibilidad de revisar incluso algunos desarrollos del marxismo en torno a las posibilidades de una crítica "moral" del capitalismo. Esta lectura de seguro no se corresponde con el proyecto teórico-político del propio Durkheim. Aún así, abre brechas de indagación nuevas sobre la regulación de las relaciones laborales en el capitalismo. Entre ellas: ¿cuál es el vínculo entre el mundo del trabajo capitalista (relación de explotación despersonalizada, des-politizada, des-moralizada, tecnificada e instrumentalizada) y la moral y la cultura popular (que ha construido significaciones y sentidos diversos, a veces contra-hegemónicos- alrededor de la condición del trabajador en la relación de mercantilización de la fuerza de trabajo)? 
Es, por [falsamente] paradójico que suene para los estudios del trabajo de la post-modernidad, en el contexto contemporáneo, en el que el mundo del trabajo atraviesa procesos de diferenciación, diversificación, fragmentación, segmentación y desestabilización más agudos, donde se manifiestan con mayor vigor prácticas, dispositivos e intervenciones de rearticulación de las esferas de lo técnico y lo normativo, del ámbito del trabajo como producción material instrumentalizada, y de la moral, como producción simbólica de reconocimiento, dignidad y legitimidad política y social ${ }^{16}$.

Debido a ciertos sesgos de ambos autores visitados (Durkheim y Habermas), las formaciones morales aparecen en distintos grados de institucionalización, dando lugar a períodos de normalidad y anomia, pero quedando completamente por fuera de las disputas y conflictos entre agentes, y por lo tanto, por fuera de cierta instrumentalizacion de la moral como recurso. Por esta razón, la conceptualización deja fuera un hecho fundamental. El análisis de la moral vinculada a los procesos y las prácticas de trabajo no debe ser realizado exclusivamente desde la perspectiva de la solidaridad social, sino también (y fundamentalmente) desde el punto de vista de la diferenciación social. La moral aparece en algunos ámbitos (fundamentalmente en aquellos en donde, por la desigual distribución de poder social, otros tipos de recursos escasean) como un instrumento simbólico fundamental de acumulación, legitimación y reclamo de reconocimiento (dignidad), y, en este sentido, de distinción entre posiciones y agentes. En alguna medida, los desarrollos de ambos autores aparecen como insuficientes para dar cuenta de la dimensión del conflicto moral.

\footnotetext{
${ }^{16}$ Sólo tuvimos espacio aquí para manifestar la actualidad de esta reflexión teórica. En una serie de textos en preparación analizaremos la manera en la que esta articulación se produce, se disputa y se apropia en el ámbito de la escolaridad para jóvenes y adultos, en políticas activas de empleo para jóvenes y en el área de recursos humanos de algunas empresas de un barrio popular del Gran Córdoba.
} 
Si en un primer movimiento la modernidad produce, junto con la tecnificación del trabajo, un vaciamiento de su contenido moral y cosmológico ${ }^{17}$; en un segundo movimiento instituye al trabajo como soporte de inscripción en la estructura social y como centro de la regulación de la cohesión social (Castel, 2000). Pero este recubrimiento moral de la vida laboral (identidad, solidaridad, ciudadanía, asistencia, etc.) puede resultar tan conservador (Martín Criado, 1999) como disruptivo respecto del orden, dependiendo del estado de fuerza y de la lucha de los actores involucrados: en el caso de las relaciones de explotación, el estado de la lucha de clases. Ya algunos autores marxistas mostraron la manera en que la resistencia a los valores y la legitimidad de la mercantilización capitalista de la vida y el trabajo se han basado en la pervivencia de valores tradicionales a la vez "conservadores" y "rebeldes" (Thompson, 1991).

Este aparece como uno de los puntos más limitados de la conceptualización de Durkheim y, en algún sentido, también de Habermas. Estos desarrollos construyen a la moralidad casi siempre como marco regulador de las prácticas laborales, pero presentan algunos problemas para dar cuenta de la manera en que esas prácticas producen contenido y estructuras morales concretas, y del modo en que estas coincidencias y/o disrupciones pueden explicarse y comprenderse. Más allá de lo que podemos entender como una "necesidad sistémica" de re-moralización y orientación de los individuos hacia el trabajo como "deber" y "condición" de reproducción social, el rumbo de dicha moralización es fundamentalmente

\footnotetext{
${ }^{17}$ En este punto sería válido preguntarse si la "economización" del mundo del trabajo se da -en la modernidad- en un sentido fundamentalmente "práctico" -es decir, en la estructuración misma de la práctica- o bien, puramente "ideológico" -siguiendo la línea de indagación en la cual incurren algunos autores (Palenzuela 1995)-, como una expresión de legitimación del simbolismo dominante en torno a lo laboral. El interrogante incluye una pregunta mayor, que es la de la posibilidad de distinguir entre estos dos niveles (el práctico y el ideológico), y que es una distinción que intenta reunificarse en el desarrollo teórico que realizamos a lo largo del artículo.
} 
contingente $y$, por lo tanto, debe ser determinado empíricamente por el investigador, antes que suponerse apriorísticamente desde la teoría. El sociólogo que intenta abordar "los elementos culturales del trabajo" como problemática requiere avanzar en el sentido de categorías que permitan dar cuenta de la moralidad como normatividad disciplinante y reguladora de la reciprocidad, pero también como recurso escaso en disputa, útil para producir desigualdad y diferencia a nivel material y simbólico.

Gonzalo Assusa - Licenciado en Sociología, Universidad Nacional de Villa María. Profesor del CONICET, Instituto de Humanidades, Centro de Investigaciones de la Facultad de Filosofía y Humanidades, Universidad Nacional de Córdoba (Argentina). $>$ gon_assusa@hotmail.com

\section{Referências}

1. ALEXANDER, J. La centralidad de los clásicos. In: GIDDENS, A.; TURNER, J. La teoría social, hoy. Madrid: Alianza Editorial, 1998.

2. BAUMAN, Z. Trabajo, consumismo y nuevos pobres. Barcelona: Gedisa, 1999.

3. CASTEL, R. La metamorfosis de la cuestión social. Una crónica del salariado. Buenos Aires: Paidós, 2000.

4. DE LA GARZA TOLEDO, E. Introducción. El papel del concepto de trabajo en la teoría social del siglo XX. In: DE LA GARZA TOLEDO, E. (Org.). Tratado latinoamericano de sociología del trabajo. México DF: El Colegio de México-Fondo de Cultura Económica-FLACSO-UAM, 2000.

5. DURKHEIM, E. El suicidio. Estudio de sociología. Buenos Aires: Centro Editor América Latina, 1994.

6. DURKHEIM, E. Lecciones de sociología. Física de las costumbres y del Derecho y otros escritos sobre el individualismo, los intelectuales y la democracia. Buenos Aires: Miño y Dávila, 2003.

7. DURKHEIM, E. La división del trabajo social. Barcelona: Planeta Agostini, 1985.

8. DROLAS, A.M. et al. Una aproximación teórica a la relación entre trabajo y cultura. Documentos del CEIL-PIETTE. Buenos Aires, 2009. Disponível em: < http:// www.grupohasa.com/cuidador/lecturas/pdf/42.pdf>. Acesso em: marzo 2012. 
9. GRIMSON, A. Los límites de la cultura. Crítica de las teorías de la identidad. Buenos Aires: Siglo XXI, 2011.

10. ELÍAS, N. El proceso de la civilización. Investigaciones sociogenéticas y psicogenéticas. México DF: Fondo de Cultura Económica, 1989.

11. EYERMAN, R.; SHIPWAY, D. Habermas on Work and Culture. In: Theory and Society. v. 10, n. 4, p. 547 - 566, jul. 1981.

12. GUADAMARRA OLIVERA, R. Cultura laboral. In: DE LA GARZA TOLEDO, E. (Org.). Tratado latinoamericano de sociología del trabajo. México DF: El Colegio de México-Fondo de Cultura Económica-FLACSO-UAM, 2000.

13. HABERMAS, J. Teoría de la acción comunicativa II. Crítica de la razón funcionalista. Madrid: Taurus, 1990.

14. HABERMAS, J. Problemas de legitimación del capitalismo tardío. Madrid: Editora Nacional Madrid, 2002.

15. HONETH, A. Teoría crítica. In: GIDDENS, A.; TURNER, J. La teoría social, hoy. Madrid: Alianza Editorial, 1998.

16. JACINTO, C.; MILLENAAR, V. Los nuevos saberes para la inserción laboral. Formación para el trabajo con jóvenes vulnerables en Argentina. In: Revista Mexicana de Investigación Educativa, México DF, v. 17, n. 52, p. 141 - 166, 2012.

17. LUKACS, G. La cosificación y la conciencia del proletariado. In: LUKACS, G. Historia y consciencia de clase. Buenos Aires: Orbis, 1985.

18. MARTÍN CRIADO, E. El paro juvenil no es el problema, la formación no es la solución. In: RODRÍGUEZ, L. (Org.). Juventudes, mercados de trabajo y políticas de empleo. Valencia: 7imig, 1999.

19. MARX, K.; ENGELS, F. La ideología alemana. Buenos Aires: Nuestra América, 2004.

20. MARX, K. Manuscritos económico-filosóficos. Buenos Aires: Colihue, 2004.

21. MEIKSINS WOOD, E. Democracia contra capitalismo. La renovación del materialismo histórico. México DF: Siglo XXI, 2000.

22. MERTON, R.K. Teoría y estructuras sociales. México DF: Fondo de Cultura Económica, 1980.

23. NEFFA, J.C.; BROWN, B.; LÓPEZ, E. Empleo, desempleo y políticas de empleo. Políticas activas de empleo durante la posconvertibilidad. 11. Ed. Tercer trimestre. Buenos Aires: CEIL-PIETTE, 2012.

24. PALENZUELA PALENCIA. Las culturas del trabajo. Una aproximación antropológica. Sociología del Trabajo, Madrid, n. 24, primavera 1995. Editorial Siglo XXI. 
25. ROCHER, G. Talcott Parsons et la Sociologie Americaine. Paris: Puf, 1972. [Capítulo II, p. 43-67. Traducción de cátedra de Alicia Gutiérrez].

26. SENNETT, R. La corrosión del carácter. Las consecuencias personales del trabajo en el nuevo capitalismo. Barcelona: Anagrama, 2000.

27. THOMPSON, E.P. Costumbres en común. Barcelona: Editorial Crítica-Grijalbo Mondadori, 1991.

28. THOMPSON, E. P. La formación de la clase obrera en Inglaterra. Barcelona: Editorial Crítica, 1989.

29. WILKIS, A.; CARENZO, S. Lidiar con bienes, lidiar con mercancías. Etnografía de transacciones económicas y morales. Apuntes de investigación del CECYP, [S.I.], n. 14, 2008.

Recebido em: 11/12/2013

Aceite final: 04/03/2014 